\title{
What's New in OJS 3
}

OJS 3 is significantly different than its predecessor, OJS 2. It includes enhancements and new features developed from community feedback, extensive usability testing, and new software design capabilities.

\section{Editorial Discussions}

To help track the communications that are a critical part of a submission's workflow, OJS 3 has a new internal discussion feature for each editorial stage (Submission, Review, Copyediting, Production). Discussions work much like an online forum - a user creates a discussion topic, invites others to participate, and sends a message (including with attachments):

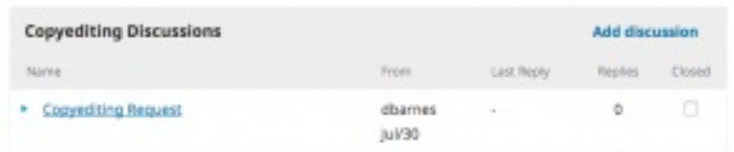

Anyone involved with the submission can be included in a discussion (the editor, section editors, authors, copyeditors, etc.). Recipients receive an email notification to inform them of the new message. Replies happen just like in a blog comment or forum post:

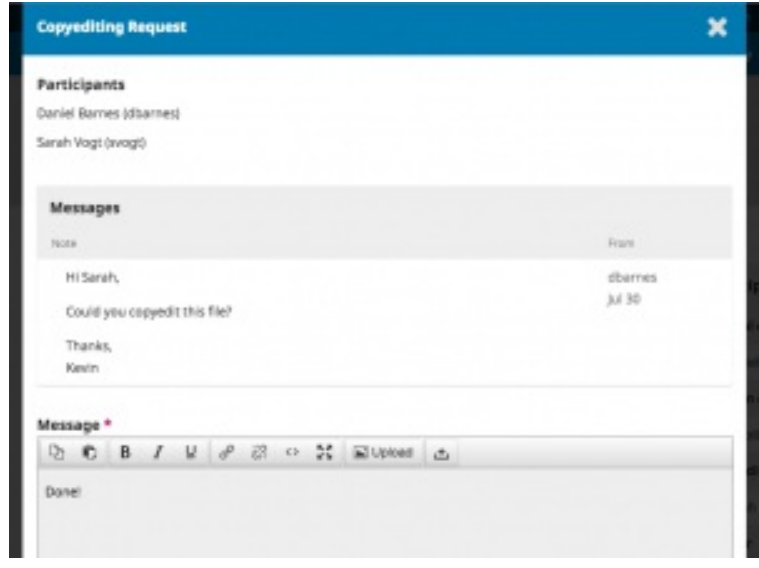

And the entire discussion thread becomes a part of the permanent editorial history.

\section{Flexible Workflow}

OJS 3 consists of 4 editorial stages: Submission, where new submissions are dealt with (rejected, assigned to section editors, etc.); Review, where peer review and author revisions take place; Copyediting, where the reviewed and revised files are sent for copyediting; and Production, where the final, copyedited version is converted into publishable formats (PDF, HTML, etc.), proofread, and scheduled for publication.

To increase editorial flexibility, you can easily move a submission from one stage to another without completing any of the possible tasks on that stage. You don't do copyediting? No problem, just skip it with the Send to Production button.

\begin{tabular}{|c|c|c|c|c|c|c|}
\hline Submission & Review & Copyediting & Production & & & (3) Help \\
\hline \multirow[t]{3}{*}{ Draft Files } & & & Q Search & Upload/Select Files & Send To Production & \multirow{3}{*}{ Add } \\
\hline & & & \multicolumn{2}{|l|}{ No Files } & \multirow{2}{*}{ Participants } & \\
\hline & & & & & & \\
\hline
\end{tabular}

You can also involve any users you wish within each stage. If your journal doesn't involve the authors in proofreading, you can simply exclude them in the discussion. 


\section{Flexible Roles}

In addition to making the workflow more flexible, you can now easily change the names of existing roles. If you'd rather have Production Managers rather than Journal Managers, you can simply rename that role.

As well, if you want to create a new role, you can do that to. OJS 3 let's you make up any role you wish, and associate with any (or all) stages of the workflow.

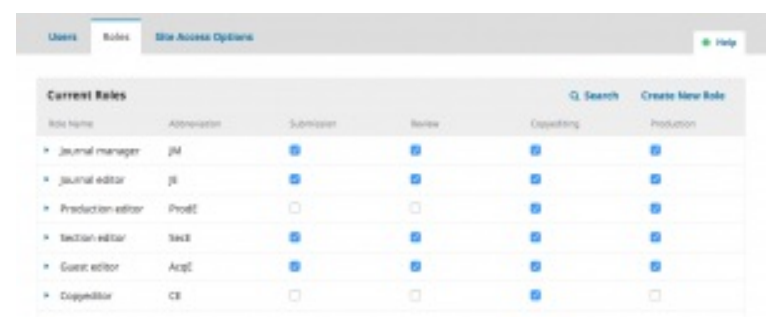

You can also configure custom roles to have similar permissions to existing roles, such as authors, reviewers, section editors, etc. For example, if you create a new Editor-in-Chief role, you could give that role access to all 4 editorial stages, and the same permissions as editors and journal managers (the ability to do anything with the journal settings and anything with submissions).

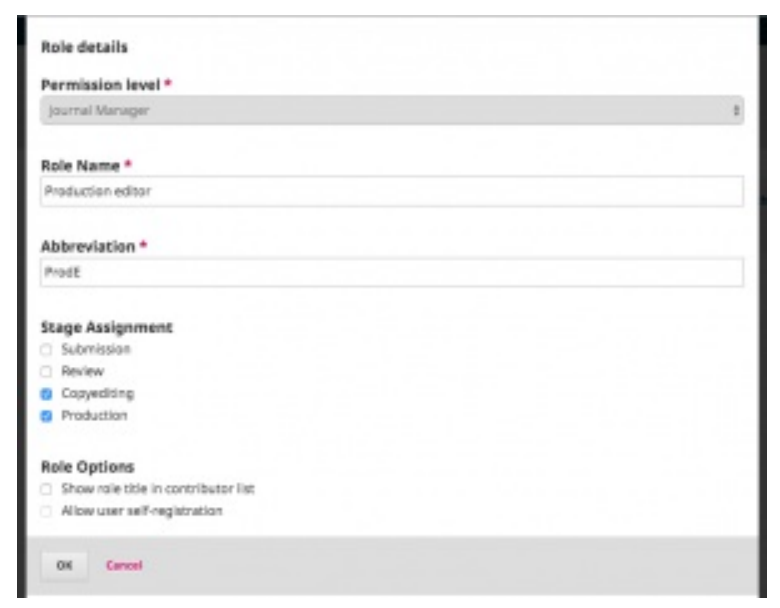

\section{Flexible Task Access}

A common annoyance with OJS 2 was the need to switch roles to do different tasks. For example, if you were a user enrolled as both a Journal Manager and an Editor, you had access to both the submissions as well as the journal settings. However, if you were in the middle of editing a submission, but realized you needed to tweak a setting on the web site, you needed to go to your user home page, select your role as Journal Manager, go to the Journal Manager home page, and then select the appropriate setting. In OJS 3, if you are logged in as a user with permission to access both the submissions and the journal settings, you no longer need to make that complicated switch. The settings are simply available in the left sidebar.

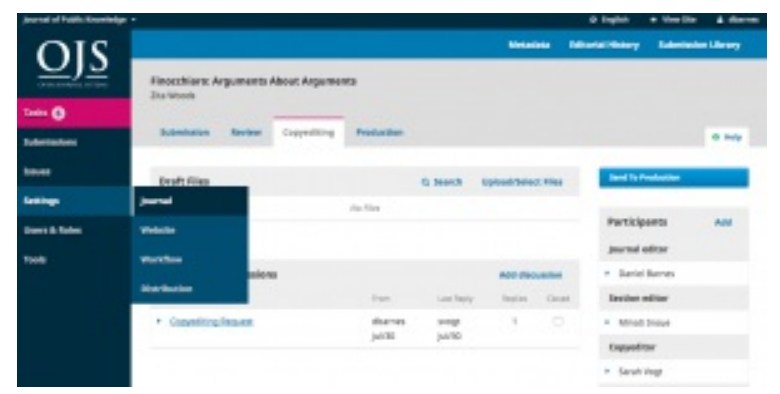

\section{Submission Files}

In OJS 2, authors can submit a single "main" file (typically the article manuscript) and then other "supplementary" files that are handled differently. In OJS 3 , the author can now upload an unlimited number of main files (article manuscript, images, tables, data sets, etc.), using the Add Another File option. 


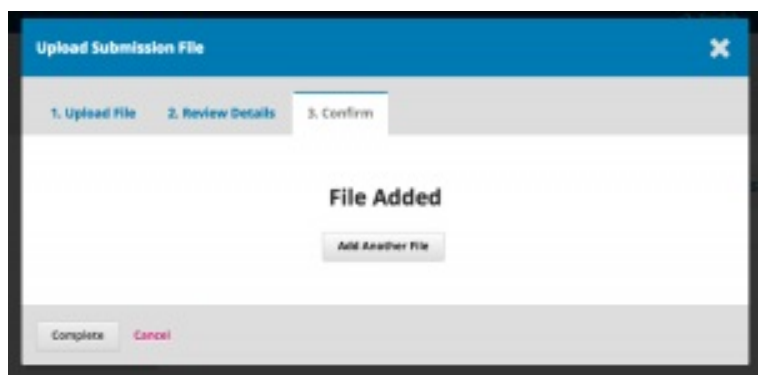

These can all be made available as part of the peer review process, as well as in the Copyediting and Production stages.

The aim here is to reduce the artificially imposed distinction between the article manuscript and any other files. For example, images of figures are central to the manuscript and should have the option of being treated as part of the main submission.

\section{Customizable Interface}

In addition to making the workflow more customizable, we have also made the user interface more flexible. In OJS 2 , creating a new theme was often a challenge due to the extensive number of style sheets that needed to be modified. To reduce this burden, we have separated the style sheets and underlying templates for the administrative interface and the reader interface.

This brings two benefits: first, users who work with multiple OJS 3 journals (e.g., as an editor for one, an author for another, and reviewer for a third) will always have the same user experience in the administrative interface — it will always look the same on the inside; second, separating out the reader interface templates and style sheets means that they will be significantly smaller and easier to modify.

\section{Bootstrap Theming}

OJS 3 allows you to upload bootstrap themes, making it easier for your journal to have a unique look.

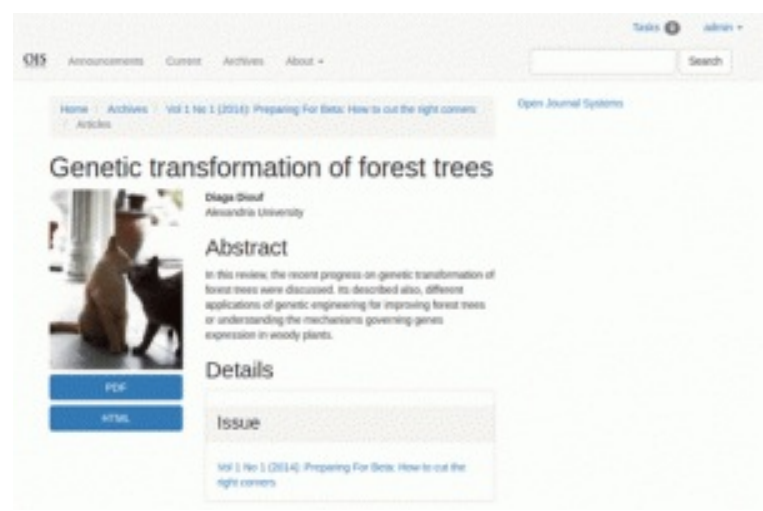

\section{Responsive Design}

Lack of responsiveness (the ability of the journal's web page to adjust to the reader's screen size — from a phone, to tablet, to desktop computer) was a major issue for OJS 2 . The OJS 3 reader interface is fully responsive.

\section{Simpler Registration}

To ease registration for new users, we've prioritized a small set of required fields on a single screen (e.g., name, affiliation, email, etc.), and allow the registration to proceed once those few are completed. 


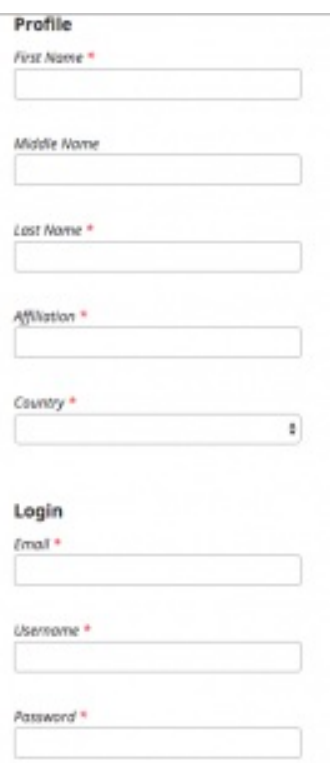

The new user can then proceed directly to make a submission or accept a review request. She has the option to fill in a more complete profile if she wishes, or can be asked by the editor to fill in details at a later point (such as upon acceptance of her submission). 